\title{
Bodily movement of approach is detected faster than that of receding
}

\author{
Hirokazu Doi • Kazuyuki Shinohara
}

Published online: 20 June 2012

(C) Psychonomic Society, Inc. 2012

\begin{abstract}
The human visual system is efficient at detecting an approaching object. In detecting approaching human beings, bodily movement serves as a cue for the visual system to compute moving direction. On the basis of this knowledge, we hypothesized that bodily movement implying approach is detected faster than receding bodily movement even when only bodily movement is available as a clue to discerning motion direction. To examine this hypothesis, we conducted a visual search experiment in which participants searched for a point-light figure with approaching or receding walking movement. Results showed that an approaching point-light figure was detected faster than a receding one. This search asymmetry was eliminated when the figures were presented upside-down. These findings indicate the potency of bodily movement that implies approach in effectively capturing visuospatial attention.
\end{abstract}

Keywords Social cognition · Visual search $\cdot$ Attention

Paying attention to an approaching object is of great importance for survival because early detection gives one additional time to prepare for the impending situation. The prioritized processing of an approaching object has been examined in seminal research by Neuhoff (1998), which revealed that human participants are more sensitive to auditory changes implying the approach, as compared with the receding, of a sound source. Likewise, previous studies have shown that an approaching object effectively captures visuospatial attention (Imura, Shirai, Tomonaga, Yamaguchi, \&

H. Doi $\cdot$ K. Shinohara $(\bowtie)$

Graduate School of Biomedical Sciences, Nagasaki University,

1-12-4 Sakamoto-cho,

Nagasaki City, Nagasaki, Japan 852-8523

e-mail: kazuyuki@nagasaki-u.ac.jp
Yagi, 2008), especially when the object is moving along a collision path (Lin, Franconeri, \& Enns, 2008).

The human perceptual system detects the approach of an object on the basis of various optical clues, such as looming (Kahan, Colligan, \& Wiedma, 2011). When the approaching object is a human being, bodily motion serves as another clue for the visual system to compute motion direction in-depth (i.e., either the person is approaching toward or receding from the viewer). It is well-known that the human visual system is quite sensitive to information about bodily movement (for a review, see Blake \& Shiffrar, 2007). A compelling illustration of this ability is recognition of a point-light display of human bodily movement first reported by Johansson (1973). Johansson attached luminescent markers to each human joint and depicted human movement as a constellation of moving point lights. Intriguingly, human adults can recognize a pointlight display as a depiction of human movement within $200 \mathrm{~ms}$ after stimulus onset (Krakowski et al., 2011). Furthermore, they can extract from it various information, such as gender (Cutting, 1978; Pollick, Kay, Heim, \& Stringer, 2005; Troje, 2002), age (Montepare \& Zebrowitz-McArthur, 1988), emotion (Atkinson, Dittrich, Gemmell, \& Young, 2004; Ikeda \& Watanabe, 2009; Pollick, Paterson, Bruderlin, \& Sanford, 2001), and walking direction (McKay, Simmons, McAleer, \& Pollick, 2009; Takahashi et al., 2011).

Considering the potency of an approaching object in attracting visuospatial attention (Imura et al., 2008; Lin et al., 2008) together with the human visual system's ability to discriminate motion direction in-depth from bodily movement (Jackson \& Blake, 2010), we hypothesized that a point-light figure with bodily movement implying approach can be spotted faster than that implying recession, even when only bodily movement is available as a cue for computing motion direction in-depth. To examine this hypothesis, we conducted a visual search experiment (Treisman \& Souther, 1985; Wolfe, 2001) in which the participants searched for a point-light figure among distractors 
of nonsensical clouds of moving dots. The main indicator of interest was the time required to detect approaching and receding point-light figures. The point-light figures were presented stereoscopically to disambiguate the in-depth location of each point light (Jackson \& Blake, 2010). If visuospatial attention is drawn to bodily movement implying approach more effectively than to that implying receding, approaching point-light figures would be detected faster than receding ones. The effect of lowlevel perceptual features was examined by measuring efficiency in detecting approaching and receding point-light figures presented upside-down. Inversion of a point-light figure is known to disrupt the vivid impression of the human figure (Pavlova \& Sokolov, 2000; Sumi, 1984; Troje \& Westhoff, 2006). Therefore, if direction of motion in-depth implied by bodily movement per se, rather than low-level perceptual features, is the main cause of predicted search asymmetry, there should be no discernible difference in search efficiency for approaching and receding point-light figures when they are presented upside-down.

\section{Method \\ Participants}

Six males and 14 females $(M=25.9$ years of age, $S D=6.8)$ with normal or corrected-to-normal visual acuity participated in this experiment.

\section{Apparatus and stimuli}

The stimuli were generated on a 19-in. color monitor, viewed binocularly from a distance of $75 \mathrm{~cm}$. Participants sat in a dark room with their heads stabilized on a chinrest. Each stimulus consisted of four point-light displays of a walking human figure or scrambled motion. The displays were created on the basis of a three-dimensional coordinate file included in a database by Vanrie and Verfaillie (2004).

The walking point-light figure showed either approaching or receding bodily movement as schematically depicted in Fig. 1a). The original point-light figure was created to look as if it was walking on a treadmill and did not appear to translate in in-depth direction. In the experiment, the figure was presented stereoscopically to disambiguate the walking direction. That is, two slightly different views of a pointlight walker with a $5^{\circ}$ orientation disparity were presented separately to each eye. The projection viewed by the right eye was presented on the display in red, while that viewed by the left eye was in green. These projections were fused to create a single three-dimensional percept of a point-light figure when viewed through the stereoscope with colored glasses. On the screen, the point-light figure subtended $8.6^{\circ}$ in height and $3.4^{\circ}$ in width at the maximum. Each point light subtended $0.3^{\circ}$ in diameter. The inverted point-light figures with approaching and receding motion were created by inverting the upright point-light displays in a vertical direction. One cycle of walking was depicted by 60 frames lasting for $1 \mathrm{~s}$. The initial frame was determined randomly.

The scrambled motions were created by randomly changing the initial position of point lights while preserving the motion vector of each. The initial position of each point light was determined by replacing it within the plane perpendicular to walking direction. The initial locations indepth of point lights were not altered to enable them to serve as an adequate control for point-light figures.

\section{Procedure}

Visual search task Two blocks of the visual search task (VST) were conducted. In the stimulus display of the VST,
Fig. 1 a Schematic representation of the concept of approaching and receding point-light figures. In the experiment, only point lights were presented without a realistically-rendered CG human model. b A sample image of the stimulus display in the Present-Upright condition. In the real experiment, slightly different views of point-light figures and scrambled motions were depicted in red and green on the stimulus display. These different views were fused to create a three-dimensional percept when viewed through the stereoscope with colored glasses a

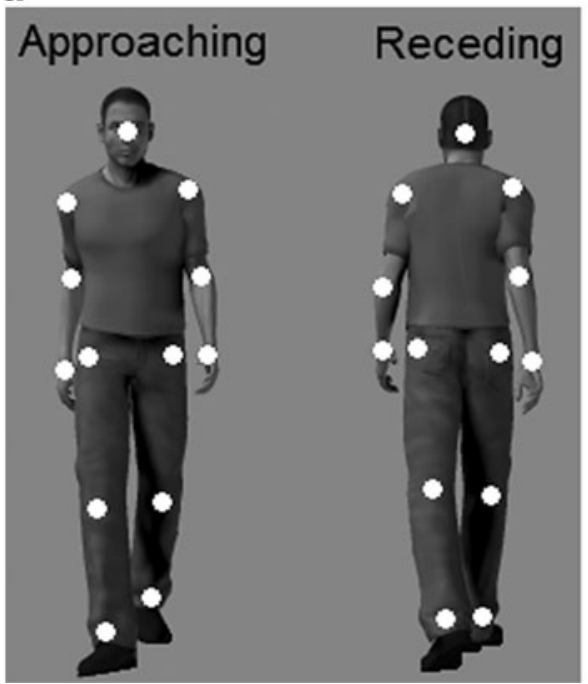

b

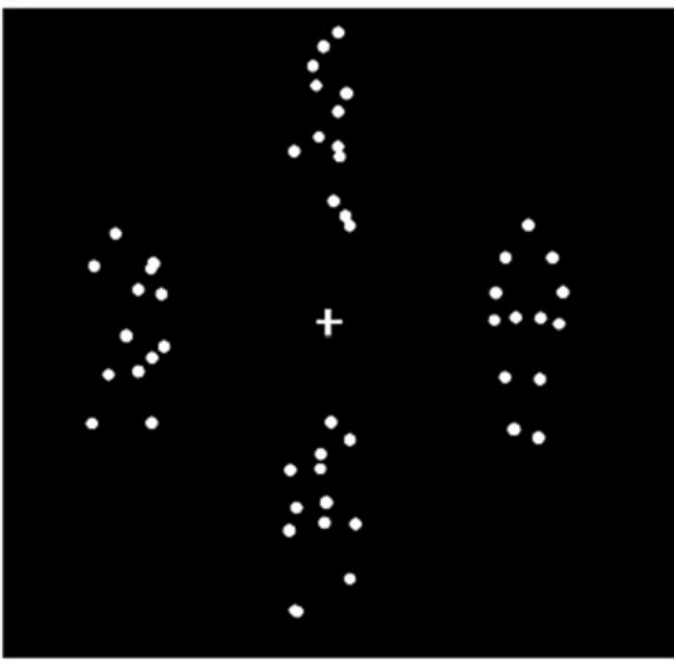


four point-light displays were presented at four locations (above, below, left, right) surrounding the fixation at the center of the screen. The distance between fixation and center of each location was kept at $5.7^{\circ}$. The stimulus display subtended $14.8^{\circ}$ in width and $19.9^{\circ}$ in height.

The VST consisted of present and absent trials. An example of a stimulus display in the present condition is shown in Fig. 1b). On present trials, orientation (upright, inverted) $\times$ direction (approaching, receding) $=$ four types of point-light figures were presented as the target among three distractors of scrambled motion. Each target appeared at each of the four locations 16 times, yielding a total of 256 present trials. Four distractors of scrambled motion were presented in the 128 absent trials conducted.

Each trial began with a fixation cross presented at the center of the display. After $500 \mathrm{~ms}$, four point-light displays were presented around the fixation cross. Both fixation cross and point-light displays were presented against a black background with $0.6 \mathrm{~cd} / \mathrm{m}^{2}$ luminance. The stimulus display remained on the screen until a response was made. Participants were asked to answer as quickly and accurately as possible whether a walking human figure, in either upright or inverted orientation, was presented in the display by pressing the "s" or "l" key. They were also instructed to fixate on the fixation cross and keep eye movements as small as possible. The correspondence between key and response was counterbalanced across participants. Immediately after the response, feedback was presented regarding participants' accuracy. The next trial started $500 \mathrm{~ms}$ after the onset of the feedback stimulus, and the whole cycle was repeated. A short break was taken between blocks.

Walking direction discrimination task To examine whether the participants could reliably discriminate the walking direction of point-light figures, a walking direction discrimination task (WDDT) was conducted after completion of the VST. In the stimulus display of the WDDT, orientation (upright, inverted) $\times$ direction (approaching, receding) $=$ four types of point-light figures were presented at the center of the screen. The participants answered the walking direction by pressing the "l" or "s" key as soon as possible. The correspondence between answer and key was counterbalanced across participants. Pressing the button triggered the next trial. Each type of point-light figure was presented 10 times, which yielded a total of 40 trials.

\section{Results}

Visual search task

Reaction time (RT) and accuracy rates were calculated for each condition. Trials with RTs shorter than $100 \mathrm{~ms}$ were discarded from final analysis as anticipatory responses. Discarded trials amounted to less than $1.6 \%$ in each condition in every participant. Averaged RT in each present condition was calculated on the basis of correct trials for each participant.

Interparticipant means of RT in each condition are depicted in Fig. 2, together with standard errors. The RTs on present trials were entered into a within-participants analysis of variance (ANOVA) with factors of orientation (upright, inverted) and direction (approaching, receding). The ANOVA revealed a significant main effect of orientation, $F(1,19)=147.9, p<.01, \eta_{\mathrm{p}}{ }^{2}=.88$, with detection of upright point-light figures being faster than that of inverted ones. The main effect of direction did not reach significance, $F(1,19)=0.67, p>.10, \eta_{\mathrm{p}}{ }^{2}=.03$. The main effect of orientation was qualified by a significant interaction between orientation and direction, $F(1,19)=5.71, p<.05$, $\eta_{\mathrm{p}}^{2}=.23$.

The simple main effect analysis revealed that approaching point-light figures were detected significantly faster than receding ones in the upright condition, $F(1,38)=5.52, p<.05$, $\eta_{\mathrm{p}}{ }^{2}=.12$, but not in the inverted condition, $F(1,38)=1.65, p>$ $.10, \eta_{\mathrm{p}}{ }^{2}=.04$. The simple main effect of orientation reached significance in both the approaching, $F(1,38)=127.3, p<.01$,
Fig. 2 Inter-participant means of RTs in each of the four Present conditions. Error bars indicate standard errors. ${ }^{*} p<.05$ for the simple main effect of walking direction

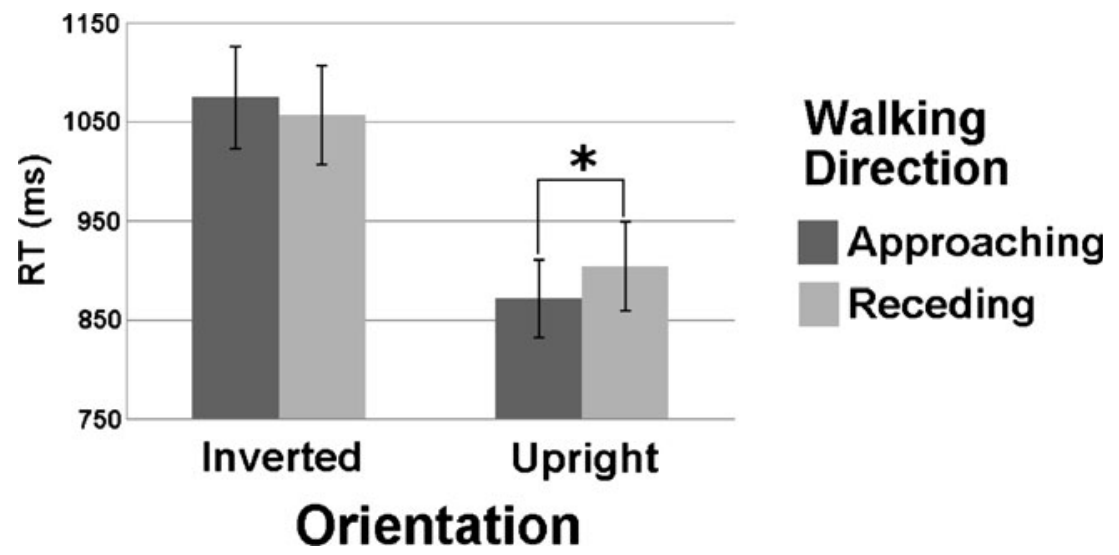


$\eta_{\mathrm{p}}^{2}=.77$, and receding, $F(1,38)=72.2, p<.01, \eta_{\mathrm{p}}{ }^{2}=.65$, direction conditions.

Accuracy rates were above $97 \%$ in every condition. They were entered into a two-way ANOVA with factors of orientation and direction. The ANOVA revealed no significant effects, $F_{\mathrm{s}}<1, p \mathrm{~s}>.10$, indicating that there was no speed-accuracy trade-off.

Walking direction discrimination task

Averaged RT and the proportion of "approaching" responses for each of orientation $(2) \times$ direction $(2)=$ four types of stimuli are summarized in Table 1 . The proportions of "approaching" responses were entered into an ANOVA with the same factorial design as that described above. The main effect of orientation failed to reach significance, $F(1,19)=0.77, p>.10, \eta_{\mathrm{p}}^{2}=.05$, but, the ANOVA revealed a significant main effect of direction, $F(1,19)=89.96, p<.01, \eta_{\mathrm{p}}{ }^{2}=.83$. These main effects were qualified by a significant interaction between orientation and direction, $F(1,19)=112.81, p<.01, \eta_{\mathrm{p}}{ }^{2}=.85$. Further analysis revealed a significant simple main effect of direction in the upright condition, $F(1,38)=191.93, p<.01$, $\eta_{\mathrm{p}}{ }^{2}=.84$, but not in the inverted condition, $F(1,38)=2.91$, $p>.10, \eta_{\mathrm{p}}{ }^{2}=.07$.

The RTs were entered into an ANOVA with the same factorial design. The ANOVA revealed a significant main effect of orientation, $(1,19)=12.49, p<.01, \eta_{\mathrm{p}}{ }^{2}=.39$, but no other effects reached significance, $F \mathrm{~s}<3.9, p \mathrm{~s}>.10$.

\section{Discussion}

The present results revealed that approaching point-light figures were detected faster than receding figures, even when the bodily movement of the figures was the only available cue for detecting motion direction in depth.

Table 1 Averaged Proportion of "Approaching" Responses and RT in each condition in Walking Direction Discrimination Task (WDDT). In the parentheses are standard deviations

\begin{tabular}{llll}
\hline & & Proportion (\%) & RT (ms) \\
\hline Inverted & Approaching & 50 & 1413.9 \\
& & $(21.1)$ & $(921.2)$ \\
& Receding & 40.5 & 1328.8 \\
& & $(22.8)$ & $(835.3)$ \\
Upright & \multirow{2}{*}{ Approaching } & 95.5 & 776.7 \\
& & $(8.3)$ & $(372.5)$ \\
& \multirow{2}{*}{ Receding } & 9.5 & 872.3 \\
& & $(2.7)$ & $(363.6)$ \\
\hline
\end{tabular}

Proportion: Proportion of “Approaching” Responses
Inverting the point-light figures eliminated this asymmetry in search efficiency, precluding the possibility that the search asymmetry was derived from low-level perceptual features. Taken together, these findings indicate the potency of approaching bodily movement in attracting visuospatial attention. Although it has previously been reported that the walking direction of a point-light figure in the lateral (leftright) plane influences attention orientation (Shi, Weng, He, \& Jiang, 2010), this is the first study to show that the observation of a point-light figure walking in the depth plane (approaching, receding) induces visual search asymmetry.

In this study, we did not investigate the effect of display size on the speed of detecting point-light figures (Treisman \& Souther, 1985; Wolfe, 2001). Thus, in principle, we cannot be certain that the observed search asymmetry constitutes an attentional phenomenon. To address this issue, we measured search efficiency for upright-approaching and upright-receding point-light figures against scrambled motion, using three display sizes (two, four, and six items) in 8 naive participants ( 5 females; $M=31.0$ years of age, $S D=$ 6.2). The procedure was identical in most respects to that used in the main experiment. The results revealed that every participant exhibited a steep increase of RT as a function of display size ( $>10 \mathrm{~ms} /$ item) for both approaching and receding point-light figures $(63.9 \pm 32.3$ and $79.1 \pm 49.4 \mathrm{~ms} /$ item for approaching and receding figures, respectively), indicating that visual search in the present study was inefficient (Wolfe, 1998). Considering this finding together with those of a number of studies indicating the necessity of active deployment of attention in searching for deviant point-light figures (Cavanagh, Labianca, \& Thornton, 2001; Hirai \& Hiraki, 2006; van Boxtel \& Lu, 2011; but see Thornton \& Vuong, 2004), it is unlikely that the observed search asymmetry was attributable to a preattentive process.

The present finding is similar to the stare-in-the-crowd effect, whereby a face with direct gaze can be detected more quickly than a face with averted gaze (Doi \& Ueda, 2007; Doi, Ueda, \& Shinohara, 2009a, 2009b; Palanica \& Itier, 2011; Senju \& Hasegawa, 2006; von Grünau \& Anston, 1995). Considering that both gaze and body orientation potentially indicate another person's locus of interest (Perrett, Hietanen, Oram, \& Benson, 1992), it is understandable that these types of information induce similar behavioral responses. Another line of research has revealed a perceptual bias for perceiving bistable point-light figures as walking toward the viewer (Schouten, Troje, Vroomen, \& Verfaillie, 2011; Vanrie \& Verfaillie, 2006). Considering that such bias reduces false negatives in detecting approaching figures, both the perceptual bias and the efficient allocation of attention toward approaching point-light figures might have evolved in the human visual system to enable efficient detection of approaching figures. 
The neural region underlying the present finding is unclear at this point. However, it seems likely that the posterior region of the superior temporal sulcus (pSTS), which is involved in decoding the intention of others (Saxe, Xiao, Kovacs, Perrett, \& Kanwisher, 2004), contributed to the observed search asymmetry. Activation of the pSTS has been observed during the processing of biological motion (Jastorff \& Orban, 2009; Saygin, Wilson, Hagler, Bates, \& Sereno, 2004; Vaina, Solomon, Chowdhury, Sinha, \& Belliveau, 2001), as well as gaze direction (Puce, Allison, Bentin, Gore, \& McCarthy, 1998; Sato, Kochiyama, Uono, \& Yoshikawa, 2009; Wicker, Michel, Henaff, \& Decety, 1998). Although the specific neural mechanism through which social information influences visuospatial attention remains contentious (Engell et al., 2010; Hietanen, Leppänen, Nummenmaa, \& Astikainen, 2008; Sato, Kochiyama, Uono, \& Yoshikawa, 2009), we posit that functional connectivity between the pSTS and frontoparietal attentional network (Corbetta \& Shulman, 2002) underlies the search asymmetry observed in the present study. However, this premise should be examined empirically in future studies.

The present results also revealed that inversion drastically delayed the detection of the point-light figure, which partially supports the contention that the human visual system is tuned to rapidly detect signals of animate forms (Troje \& Westhoff, 2006). This result is in stark contrast with the findings of Wang, Zhang, He, and Jiang (2010), who reported no difference in efficiency in searching for upright and inverted point-light figures with intact global configuration. We propose that this discrepancy was derived from differences in experimental procedures between the two studies. In their study, inverted point-light figures served as distractors in the visual search for an upright point-light figure, and vice versa.

Finally, limitations of the present study should be considered. Bülthoff, Bülthoff, and Sinha (1998) reported that bottom-up depth information can be overridden by topdown information extracted from point-light figures. Considering this finding together with the human visual system's bias to perceive point-light figures as facing toward the viewer (Vanrie \& Verfaillie, 2006), receding point-light figures might have been perceived as having a scrambled in-depth structure due to the conflict between top-down perceptual bias and bottom-up stereoscopic depth information. However, the participants were able to recognize the walking direction of upright point-light figures fairly accurately, which partially refutes this possibility. Nevertheless, the perceptual representation of receding point-light figures should be clarified in more detail in future studies to further test the validity of the present findings.

In summary, the present study revealed that approaching point-light figures can be detected faster than receding figures. Because approaching implies an intention to interact with the observer, the predisposition to rapidly allocate visuospatial attention toward approaching figures might have evolved to cope effectively with upcoming situations.

\section{References}

Atkinson, A. P., Dittrich, W. H., Gemmell, A. J., \& Young, A. W. (2004). Emotion perception from dynamic and static body expressions in point-light and full-light displays. Perception, 33, 717746.

Blake, R., \& Shiffrar, M. (2007). Perception of human motion. Annual Review of Psychology, 58, 47-73.

Bülthoff, I., Bülthoff, H., \& Sinha, P. (1998). Top-down influences on stereoscopic depth-perception. Nature Neuroscience, 1, 254-257.

Cavanagh, P., Labianca, A. T., \& Thornton, I. M. (2001). Attentionbased visual routines: Sprites. Cognition, 80, 47-60.

Corbetta, M., \& Shulman, G. L. (2002). Control of goal-directed and stimulus-driven attention in the brain. Nature Reviews Neuroscience, 3, 201-215.

Cutting, J. E. (1978). Generation of synthetic male and female walkers through manipulation of a biomechanical invariant. Perception, 7 , 393-405.

Doi, H., \& Ueda, K. (2007). Searching for a perceived stare in the crowd. Perception, 36, 773-780.

Doi, H., Ueda, K., \& Shinohara, K. (2009a). Neural correlates of the stare-in-the-crowd effect. Neuropsychologia, 47, 1053-1060.

Doi, H., Ueda, K., \& Shinohara, K. (2009b). Relational property between head and eye regions is the primary determinant of the efficiency in search for a deviant gaze. Quarterly Journal of Experimental Psychology, 62, 1723-1737.

Engell, A. D., Nummenmaa, L., Oosterhof, N. N., Henson, R. N., Haxby, J. V., \& Calder, A. J. (2010). Differential activation of frontoparietal attention networks by social and symbolic spatial cues. Social Cognitive and Affective Neuroscience, 5, 432-440.

Hietanen, J. K., Leppänen, J. M., Nummenmaa, L., \& Astikainen, P. (2008). Visuospatial attention shifts by gaze and arrow cues: An ERP study. Brain Research, 1215, 123-136.

Hirai, M., \& Hiraki, K. (2006). Visual search for biological motion: An event-related potential study. Neuroscience Letters, 403, 299304.

Ikeda, H., \& Watanabe, K. (2009). Anger and happiness are linked differently to the explicit detection of biological motion. Perception, 38, 1002-1011.

Imura, T., Shirai, N., Tomonaga, M., Yamaguchi, M. K., \& Yagi, A. (2008). Asymmetry in the perception of motion in depth induced by moving cast shadows. Journal of Vision, 8 (Art. 10).

Jackson, S., \& Blake, R. (2010). Neural integration of information specifying human structure from form, motion, and depth. Journal of Neuroscience, 30, 838-848.

Jastorff, J., \& Orban, G. A. (2009). Human functional magnetic resonance imaging reveals separation and integration of shape and motion cues in biological motion processing. Journal of Neuroscience, 29, 7315-7329.

Johansson, G. (1973). Visual perception of biological motion and a model for its analysis. Perception \& Psychophysics, 14, 201-211.

Kahan, T. A., Colligan, S. M., \& Wiedman, J. N. (2011). Are visual features of a looming or receding object processed in a capacityfree manner? Consciousness and Cognition, 20, 1761-1767.

Krakowski, A. I., Ross, L. A., Snyder, A. C., Sehatpour, P., Kelly, S. P., \& Foxe, J. J. (2011). The neurophysiology of human biological motion processing: A high-density electrical mapping study. Neurolmage, 56, 373-383. 
Lin, J. Y., Franconeri, S., \& Enns, J. T. (2008). Objects on a collision path with the observer demand attention: Research article. Psychological Science, 19, 686-692.

McKay, L. S., Simmons, D. R., McAleer, P., \& Pollick, F. E. (2009). Contribution of configural information in a direction discrimination task: Evidence using a novel masking paradigm. Vision Research, 49, 2503-2508.

Montepare, J. M., \& Zebrowitz-McArthur, L. (1988). Impressions of people created by age-related qualities of their gaits. Journal of Personality and Social Psychology, 55, 547-556.

Neuhoff, J. G. (1998). Perceptual bias for rising tones. Nature, 395, $123-124$.

Palanica, A., \& Itier, R. J. (2011). Searching for a perceived gaze direction using eye tracking. Journal of Vision, 11, 1-13.

Pavlova, M., \& Sokolov, A. (2000). Orientation specificity in biological motion perception. Perception \& Psychophysics, 62, 889899.

Perrett, D. I., Hietanen, J. K., Oram, M. W., \& Benson, P. J. (1992). Organization and functions of cells responsive to faces in the temporal cortex. Philosophical transactions of the Royal Society $B, 335,23-30$.

Pollick, F. E., Kay, J. W., Heim, K., \& Stringer, R. (2005). Gender recognition from point-light walkers. Journal of Experimental Psychology. Human Perception and Performance, 31, 12471265.

Pollick, F. E., Paterson, H. M., Bruderlin, A., \& Sanford, A. J. (2001). Perceiving affect from arm movement. Cognition, 82, B51-B61.

Puce, A., Allison, T., Bentin, S., Gore, J. C., \& McCarthy, G. (1998). Temporal cortex activation in humans viewing eye and mouth movements. Journal of Neuroscience, 18, 2188-2199.

Sato, W., Kochiyama, T., Uono, S., \& Yoshikawa, S. (2009). Commonalities in the neural mechanisms underlying automatic attentional shifts by gaze, gestures, and symbols. NeuroImage, 45 , 984-992.

Saxe, R., Xiao, D.-K., Kovacs, G., Perrett, D. I., \& Kanwisher, N. (2004). A region of right posterior superior temporal sulcus responds to observed intentional actions. Neuropsychologia, 42, $1435-1446$.

Saygin, A. P., Wilson, S. M., Hagler, D. J., Jr., Bates, E., \& Sereno, M. I. (2004). Point-light biological motion perception activates human premotor cortex. Journal of Neuroscience, 24, 6181-6188.

Schouten, B., Troje, N. F., Vroomen, J., \& Verfaillie, K. (2011). The effect of looming and receding sounds on the perceived in-depth orientation of depth-ambiguous biological motion figures. PLoS ONE, 6(Art.e14725).
Senju, A., \& Hasegawa, T. (2006). Do the upright eyes have it? Psychonomic Bulletin \& Review, 13, 223-228.

Shi, J., Weng, X., He, S., \& Jiang, Y. (2010). Biological motion cues trigger reflexive attentional orienting. Cognition, 117, 348-354.

Sumi, S. (1984). Upside-down presentation of the Johansson moving light-spot pattern. Perception, 13, 283-286.

Takahashi, K., Fukuda, H., Ikeda, H., Doi, H., Watanabe, K., Ueda, K., \& Shinohara, K. (2011). Roles of the upper and lower body in direction discrimination of point-light walkers. Journal of Vision, $11,1-13$.

Thornton, I. M., \& Vuong, Q. C. (2004). Incidental processing of biological motion. Current Biology, 14, 1084-1089.

Treisman, A., \& Souther, J. (1985). Search asymmetry: A diagnostic for preattentive processing of separable features. Journal of Experimental Psychology. General, 114, 285-310.

Troje, N. F. (2002). Decomposing biological motion: A framework for analysis and synthesis of human gait patterns. Journal of Vision, 2, 371-387.

Troje, N. F., \& Westhoff, C. (2006). The inversion effect in biological motion perception: Evidence for a "life detector"? Current Biology, 16, 821-824.

Vaina, L. M., Solomon, J., Chowdhury, S., Sinha, P., \& Belliveau, J. W. (2001). Functional neuroanatomy of biological motion perception in humans. Proceedings of the National Academy of Sciences, 98, 11656-11661.

van Boxtel, J. J. A., \& Lu, H. (2011). Visual search by action category. Journal of Vision, 11, 1-14.

Vanrie, J., \& Verfaillie, K. (2004). Perception of biological motion: A stimulus set of human point-light actions. Behavior Research Methods, Instruments, \& Computers, 36, 625-629.

Vanrie, J., \& Verfaillie, K. (2006). Perceiving depth in point-light actions. Perception \& Psychophysics, 68, 601-612.

von Grünau, M., \& Anston, C. (1995). The detection of gaze direction: A stare-in-the-crowd effect. Perception, 24, 1297-1313.

Wang, L., Zhang, K., He, S., \& Jiang, Y. (2010). Searching for life motion signals: Visual search asymmetry in local but not global biological-motion processing. Psychological Science, 21, 10831089.

Wicker, B., Michel, F., Henaff, M.-A., \& Decety, J. (1998). Brain regions involved in the perception of gaze: A pet study. NeuroImage, 8, 221-227.

Wolfe, J. M. (1998). Visual search. In H. Pashler (Ed.), Attention (pp. 13-73). London: University College London Press.

Wolfe, J. M. (2001). Asymmetries in visual search: An introduction. Perception \& Psychophysics, 63, 381-389. 